\title{
El rol del docente investigador en Odontología
}

\section{The role of the professor researcher in Dentistry}

\section{Resumen}

La educación odontológica en la actualidad presenta retos importantes tanto para la universidad como para los docentes ya que debido a los cambios que el mundo afronta, debe cumplirse más que nunca con los tres pilares de la educación universitaria: docencia, investigación y extensión. Ya no es suficiente la simple trasmisión de conocimientos por parte del docente sino la exposición del estudiante a la realidad social a través de la extensión, más aún en carreras científicas como la Odontología. Por último, el docente tiene la obligación de formar al estudiante como investigador para que este sea capaz de generar conocimientos y con ellos dar respuestas a problemáticas sociales. Para ello, el profesor debe contar con ciertos rasgos y desarrollar un perfil que le permita a su vez estar en la capacidad de educar a los estudiantes en el área de investigación, para ello es fundamental que el docente sea un ejemplo de producción intelectual, así como estar al día con el manejo de las nuevas tecnologías como las redes sociales científicas.

Palabras clave: Capacitación; Conocimientos; Docente; Investigación.
ISSN: 1560-9111

\section{Artículo de Revisión}

\author{
Mariana Morales-Chávez ${ }^{1, a}$ \\ ${ }^{1}$ Centro de Investigaciones Odontológicas. Uni- \\ versidad Santa María. Caracas, Venezuela. \\ a Odontopediatra. PhD en Odontología

\section{Correspondencia: \\ Mariana Morales-Chávez} \\ Correo electrónico: macamocha@hotmail.com \\ Avenida Trinidad con calle Caracas. Centro \\ Profesional Vizcaya. Piso 3. Of. 3-7. Caracas, \\ Venezuela.
}

Conflicto de intereses: La autora declara no tener conflictos de interés.

Fuente de financiamiento: Ninguno.

Fecha de recepción: 23/09/17

Fecha de aceptación: 16/10/17

\begin{abstract}
Nowdays, academic instruction in any Dental school presents important challenges for both the university and professors. Due to the constant changes that the world faces; now more than ever the three pillars of university education: teaching, research and extension, must be fulfilled. The mere transmission of knowledge by the professor is no longer enough, equal importance has the student's exposure to social reality through extension, even more in scientific careers such as Dentistry. With that purpose, professors have the obligation to train the student as a researcher so that they are able to generate knowledge and provide answers to social problems. Hence, the professor must have certain features and develop a profile that allows him to transmit research skills. It is essential that the professor should be an example of intellectual production, as well as being up to date with the management of new technologies in scientific and social networks.
\end{abstract}

Keywords: Knowledge; Professor; Research; Training.

( ) Los autores. Este artículo es publicado por la revista Odontología Sanmarquina de la Facultad de Odontología, Universidad Nacional Mayor de San Marcos. Este es un artículo de acceso abierto, distribuido bajo los términos de la licencia Creative Commons Atribucion - No Comercia_Compartir Igual 4.0 Internacional. (http://creativecommons.org/licenses/by-nc-sa/4.0/) que permite el uso no comercial, distribución y reproducción en cualquier medio, siempre que la obra original sea debidamente citada. 


\section{Introducción}

En América Latina, la investigación científica y el avance tecnológico exhiben un desarrollo muy reciente, debido a un amplio conjunto de factores políticos, económicos así como institucionales que han obstaculizado su alcance. En efecto, en Latinoamérica la investigación científica tuvo un desarrollo tardío, salvo en países como Argentina, Brasil y México; pues es sólo a partir de los años cincuenta, cuando se inicia la formulación de políticas que originaron la institucionalización de la investigación científica y tecnológica y de distintos mecanismos de desarrollo en el sector ${ }^{1,2}$.

La investigación científica es un motor fundamental en el campo de la salud y la protección social y un mejor uso del conocimiento científico podría mejorar considerablemente el nivel de salud de las sociedades. En este sentido, las universidades son el espacio por excelencia para originar las investigaciones, ya que su función principal es la generación de nuevos conocimientos que ayuden a transformar sus entornos ${ }^{3}$.

En el mismo orden de ideas, la educación odontológica está directamente ligada a la investigación científica ya que permite generar nuevos conocimientos para incrementar la calidad del ejercicio profesional y rectificar conocimientos previos que van de la mano de los avances científicos y tecnológicos, es por ello, que la investigación debe constituir la tarea principal de la universidad, lo cual incluye una búsqueda motivada y direccionada que desarrolla el conocimiento; utilizando herramientas como la observación, la experimentación, la indagación del pasado y también de la razón. Es por ello, que la investigación representa una de las actividades primordiales para el perfeccionamiento de la docencia; pues ella constituye la interrelación en el quehacer del proceso enseñanza-aprendizaje ${ }^{4,5}$.

En este artículo se hace una revisión de la literatura que busca analizar el perfil del docente desde su enfoque como investigador en las facultades de odontología. La necesidad de esta investigación radica en que las escuelas de odontología deben adaptarse a los nuevos programas educativos mundiales donde la producción de conocimientos es uno de los objetivos primordiales que busca formar al estudiante con la capacidad de crear e innovar para así generar respuestas a las problemáticas de salud bucal de la sociedad y por ende, el docente juega un rol protagónico en dicha tarea.

El objetivo del presente estudio es analizar el rol del docente en la formación de los estudiantes de Odontología como investigadores.

\section{El docente investigador}

En la actualidad, diversos autores indican que investigar demanda la existencia de un sujeto con una serie de particularidades, entre estos se encuentra Spengler ${ }^{6}$, quien afirma que la determinación de las funciones docentes hoy en día es muy diferente a la de la docencia convencional. En efecto, el docente suele recibir una trilogía de órdenes: que van desde la preparación de profesio- nales competentes para el mercado laboral; pasando por la formación de estudiantes que busquen la verdad mediante la investigación; y por último, proyectar el saber en el escenario social. Es por ello, que se plantea que la profesión docente está conformada por tres rasgos centrales; el saber técnico-pedagógico, el saber disciplinar y el compromiso moral con la comunidad ${ }^{7}$.

Hernández ${ }^{8}$ menciona que la docencia y la investigación como funciones primordiales de la universidad deben estar inclinadas hacia la extensión, con el objetivo de proyectarse a la comunidad para que esta se beneficie de la labor y de los resultados de planes, programas y proyectos que realiza. Por lo tanto, es responsabilidad del profesor universitario que el aprendizaje del estudiante sea significativo, es decir, que el estudiante asuma una conducta crítica, académica, tecnológica y humana tanto dentro como fuera de las aulas.

El profesor e investigador alemán Jaspers ${ }^{9}$, planteaba que el docente investigador es quien pone al estudiante en contacto directo con el proceso del conocimiento en sí y, es el contacto con el que se contempla la ciencia originariamente. Según Hernández ${ }^{5}$, el docente universitario que lleva a cabo y supervisa procesos investigativos con otros docentes y estudiantes, tiene en sus manos la invaluable labor de ser creador y constructor de conocimiento a través de la experiencia directa y sistematizada, que le permiten cada una de las etapas de la investigación y los resultados que la misma genera. En el mismo orden de ideas, Hernández ${ }^{8}$ afirma que "el docente que investiga y enseña desde su propia experiencia de conocer, tiene la práctica originaria y secuencial del desarrollo del saber, que le permite orientar la formación del espíritu científico e innovador de sus discípulos”.

Cuando se habla del docente universitario que vincula la función investigativa a su ejercicio profesional, se puede asegurar que él mismo está en constante creación, construyendo y reconstruyendo saberes en el desarrollo de la actividad académica en el aula, en prácticas de investigación y extensión a la comunidad, dando respuesta a los tres pilares fundamentales de la universidad ${ }^{5}$.

Es por ello, que como lo plantean Pirela y Prieto ${ }^{10}$, los docentes como investigadores deben tener o desarrollar competencias específicas, teniendo principal interés en el dominio de la terminología, los procesos metodológicos e investigativos y las teorías más importantes del campo de la investigación, con fundamento científico, que le permita acercarse de manera crítica a la realidad, utilizando la capacidad de análisis y síntesis, para poder generar en este sentido nuevos conocimientos.

En ese mismo orden de ideas, se describe al docente investigador como un intelectual reflexivo y crítico, con capacidad de transformar su propia realidad, comprometido con la sociedad. Asimismo, una de sus principales características es fomentar en el estudiante el pensamiento crítico y creativo que le permita realizar planteamientos de problemas y búsqueda de soluciones enmarcadas en el método científico. Es por ello, que el docente investigador es realmente quien participa efi- 
cazmente en la formación de profesionales competentes y exitosos ${ }^{8}$.

En el mismo contexto, Hernández ${ }^{8}$ asevera que el docente investigador es más consciente que otros docentes, de la transformación y el avance que sufre el conocimiento; ya que a través de la investigación está en constante búsqueda para consolidar el proceso de aprendizaje de sus estudiantes mediante la construcción y consolidación de la ciencia.

Todo docente responsable de la formación de profesionales de pregrado, en cualquier área del saber, debe orientar su praxis hacia el logro de valores y competencias según los requerimientos y las necesidades actuales. En ese sentido, la formación universitaria está conformada por tres roles fundamentales en la vida del ser humano: la formación como persona, la formación como ciudadano y la formación como profesional para su inserción en el campo laboral. Lamentablemente en la actualidad muchos docentes centran su enseñanza solo en la formación profesional haciendo de los estudiantes seres competitivos más no críticos y agudos de pensamiento. En otras palabras, el docente debe ser integral para educar a profesionales integrales, que se formen académicamente, como seres humanos y que sepan llevar a cabo investigaciones y labores de extensión ${ }^{7}$.

En el caso de las facultades de odontología ese cambio de paradigma en la integración de la investigación a la enseñanza es muy importante ya que se están formando profesionales en el área de la salud que deben estar capacitados para reconocer la mejor evidencia científica para proponer el tratamiento más adecuado para hacer frente a las enfermedades bucales que se presenten en las comunidades adyacentes a la universidad, que pueden variar de una zona a otra y de una población a otra, en función de las particularidades presentes en dichas poblaciones. Es por ello, que los estudios epidemiológicos son vitales para conocer la población con la que tienen contacto y de esa manera, estar mejor capacitados para darle soluciones en el marco de la rigurosidad científica. Asimismo, otros tipos de investigaciones como las revisiones bibliográficas, reportes de casos clínicos, estudios de prevalencia, entre otros, harán que los estudiantes de pregrado puedan estar mejor capacitados para dar respuestas efectivas a la comunidad ${ }^{11,12}$.

\section{Formación del docente investigador}

Es interesante acotar que el ideal de la universidad alemana fue integrar la investigación a la labor docente, lo que la convirtió en motor del desarrollo de la sociedad. Desde ese concepto, se entiende lo minucioso que debe ser la selección de los docentes universitarios ya que no deben ser solo un ente trasmisor de conocimientos que ni ellos mismos han logrado asimilar a profundidad. Según el criterio de la universidad alemana, los docentes vinculados a la universidad deben investigar, buscar y producir ciencia ${ }^{13}$.

Sin embargo, para que dichas competencias puedan ser adquiridas por el docente, éste debe ser capacitado.
Es por ello, que una de los principales objetivos de la universidad debe ser invertir en la formación de recurso humano calificado en posgrados de alto nivel donde la investigación esté considerada como un ingrediente importante. Es decir, no solo se debe hacer énfasis en que el profesor cuente con alguna especialidad del área odontológica, sino que durante su posgrado haya sido preparado en investigación y la haya puesto en práctica. Por ende, lo ideal es que los docentes sean capacitados en diferentes universidades para poder crear un cuadro académico con visiones diferentes y complementarias. Otra área importante que el docente debe dominar es el idioma inglés, ya que la mayoría de los órganos de divulgación en el área de las ciencias de la salud en la actualidad publican en ese idioma ${ }^{14}$.

Morales et al. ${ }^{15}$ afirman que todos los profesores universitarios, con su ejemplo, con su práctica, pueden contribuir con la formación de los estudiantes en la investigación ya que vivir la experiencia como investigador fortalece su formación como docente en esa área y le da la oportunidad de orientar todo el proceso más eficazmente, ya que las experiencias personales exitosas pueden extrapolarse al salón de clases. El hecho de compartir experiencias de lo que el docente ha investigado en el pasado, los proyectos que desarrolla en la actualidad y los planes investigativos futuros que tiene, serán sin duda una importante fuente de aprendizaje para el estudiante de pregrado, pues de esa forma se genera un espíritu favorable a la investigación, promoviendo esta importante práctica. De otra forma, si el docente de investigación no hace investigación, debe cambiar sus prácticas y comenzar a realizar estudios en su área específica de conocimiento para poder ser realmente un modelo positivo para el estudiante.

En ese sentido, en muchas universidades se observa que los docentes que imparten las materias relacionadas con investigación, no realizan ni publican trabajos. Así lo confirman Cruz et al. ${ }^{16}$ quienes luego de realizar un estudio en una facultad de odontología en Honduras, determinaron que la mayoría de los docentes no estaban involucrados en proyectos de investigación durante los últimos cinco ańos. Esto suele verse replicado en el área. La mayoría de ellos no cuenta con la experiencia y capacitación en investigación por lo que evade hacerlo, dividiendo de esa manera el binomio docente - investigador.

Esta realidad se ve reflejada en la producción intelectual, según Fog ${ }^{17}$ solo cuatro países de América Latina y el Caribe producen casi el $90 \%$ de la ciencia universitaria y casi la mitad de las universidades del continente no investigan ni publican suficientemente. Ayala ${ }^{2}$ concluyó que la participación de América Latina en la producción de conocimiento científico no alcanza el $1 \%$ del total mundial, sin embargo, contribuye en más de un $20 \%$ de la producción de los llamados países del tercer mundo.

\section{Incentivos para el docente investigador}

Pareciera estar muy claro que la docencia y la investigación siempre deben ir de la mano. Sin embargo, se ha demostrado a lo largo de los ańos, sobre todo en uni- 
versidades de los países en vías de desarrollo que solo los profesores que tienen un contrato de tiempo completo, que por lo general se otorga a aquellos que posean un doctorado o, por otro lado, aquellos que hayan disfrutado de una beca de formación en investigación, son los que tienen experiencia como investigadores. En el segundo caso, pueden tener más preparación para la realización y gestión de proyectos de investigación. Sin embargo, debido a lo bajo que suelen ser los salarios, se dificulta el hecho de que se concreten los proyectos de investigación ${ }^{18}$.

Dicha realidad desmotiva a los profesores universitarios, quienes en su mayoría deciden dedicarse a la docencia de forma aislada sin considerar que docencia e investigación deben ir de la mano, e incluso muchos de los profesores de la planta docente universitaria deberían dedicar el cien por ciento de sus horas laborales en la universidad a la investigación, ya que esta es la única manera de que se logren alcanzar mejores resultados y una mayor cantidad de publicaciones anualmente ${ }^{19}$.

Para luchar en cierta medida contra la desmotivación de los docente investigadores, a partir de mediados de los años 80, distintos gobiernos latinoamericanos comenzaron a aplicar políticas para promover la investigación científica y tecnológica a nivel nacional, mediante la creación de programas de incentivos económicos individuales para científicos y profesores universitarios por la realización de un conjunto de actividades y la adquisición de ciertas credenciales principalmente en el área de investigación. Sin embargo, esos incentivos suelen ser bajos y no representan un ingreso significativo para los docentes ${ }^{20}$.

\section{Características del docente investigador}

Campos ${ }^{21}$ plantea la existencia de ciertos rasgos que un docente investigador debe poseer para un desempeño adecuado de sus labores universitarias. Entre ellas, se destacan el poder para ayudar al estudiante, la entrega de sus propios ojos, sus vivencias, sus conocimientos para que los estudiantes investiguen la realidad con ahínco y profundidad, en un afán de entenderla, sin condenarla, y así buscar las acciones pertinentes para mejorarla. Aunado a ello, la lucha contra la rutina, el aburrimiento, el adormecimiento para facilitar la adquisición de conocimientos, el compartir con los miembros de la comunidad educativa sus construcciones científicas y sus vivencias, al publicarlas, discutirlas en foros, debates u otras actividades de esta naturaleza. Así como la formación con otros docentes investigadores de grupos de pensamiento y la elaboración de tendencias educativas pertinentes a su contexto.

Por otro lado, algunas de las estrategias que Morales et al. ${ }^{15}$ plantean como referencia para la enseñanza de la investigación en las aulas universitarias son: leer investigaciones publicadas sobre áreas afines a la que se esté investigando para el momento; realizar exposiciones conceptuales sobre el proceso de investigación, visto de manera global, como un sistema, a través del método científico; acompañar al estudiante investigador en el proceso de investigación mediante asesorías, lecturas y reuniones; en otras palabras, enseñar a investigar investigando o enseñar con el ejemplo. Es importante también la existencia de una relación asertiva entre el tutor y el estudiante investigador en el proceso de investigación, el investigar en y con la comunidad, mediante la divulgación de información sobre las líneas de investigación.

En efecto, la investigación propia puede convertirse en un ejemplo que debe ser llevado al salón de clases, a los laboratorios o a la comunidad para darle utilidad y desarrollar junto a los estudiantes su interés y capacidad para investigar. En otras palabras, sólo un docente que realmente investigue tendrá la credibilidad, por su experiencia, para la enseñanza de la investigación ya que esa misma experiencia le dará la sabiduría para enseñar ${ }^{15}$. En ese mismo sentido, Roig ${ }^{22}$ asevera que en la educación universitaria, la interacción profesor-alumno es indispensable durante la formación en investigación, a pesar que los modelos educativos no siempre permiten ese binomio.

El uso de tecnologías como la Internet constituye una herramienta muy valiosa para que la investigación científica tenga diversas posibilidades para su desarrollo, que implican nuevos conocimientos, habilidades y actitudes científicas por parte de los investigadores y que requieren esfuerzos educativos en esta línea. Todas las posibilidades de comunicación a través de Internet permiten el intercambio de opiniones y conocimientos entre estudiantes y profesores lo cual fomenta el desarrollo de habilidades científicas, creativas y expresivas. En ese sentido, actualmente se maneja el concepto de las redes sociales científicas, las cuales son herramientas de comunicación que pueden ser de gran utilidad para relacionarse entre investigadores, así como para la descarga y divulgación de investigaciones ${ }^{23-26}$.

\section{Conclusiones}

El rol del docente en la capacitación de los estudiantes de Odontología como investigadores es fundamental pues éste es el encargado de enseñar al estudiante, principalmente a través de su ejemplo y mentoría. Es por ello que éste debe contar con características específicas que permitan dicha formación, por lo que se hace muy importante que las universidades sean exigentes en los procesos de selección y que se invierta en la formación de docentes investigadores en diferentes universidades que permitan crear un cuerpo académico diverso para que de esa forma cuenten con herramientas en el área de la investigación. Asimismo, es indispensable que las universidades y los países se replanteen los incentivos económicos de los docentes para que estos tengan mayor motivación y además de investigar se dediquen a publicar sus estudios en medios internacionales de divulgación científica.

\section{Referencias bibliográficas}

1. Huggett S. The Rise of Latin American Science. Country Trends. 2012;31:1-3.

2. Ayala FJ. Science in Latin America. Science. 1995;267:826-827. 
3. Álvarez Echeverri JC, Echeverri Jiménez G. Investigar para publicar: una pregunta y una propuesta para la escritura de los docentes en la universidad. Unipluriversidad. 2012;5(2):51-60.

4. Cazau P. La mportancia de la investigación en la formación del odontólogo. RAAO. 2013;51(2):63-66.

5. Hernández Arteaga I. El docente investigador en la formación de profesionales. Rev Virtual Univer Cat Norte. 2009;27:1-21.

6. Spengler M, Egidi L, Craveri A. (2007). El nuevo papel del docente universitario: el profesor colectivo. Departamento de Matemática, Escuela de Estadística. Universidad Nacional de Rosario. Undécimas Jornadas "Investigaciones en la facultad" de Ciencias Económicas y Estadística; 2006.

7. Contreras Domingo J. La autonomía del profesorado. Barcelona: Morata;1995.

8. Hernández Arteaga I. El docente investigador como creador de conocimiento. Umbaga. 2009;1(4):185-198.

9. Jaspers K. La idea de la Universidad. Traducción: Agustina Schroeder. En: La idea de la universidad en Alemania. Buenos Aires: Editorial Sudamericana; 1946.

10. Pirela L, Prieto L. Perfil de competencias del docente en la función de investigador y su relación con la producción intelectual. Opción. 2006;22(50):159-177.

11. Morita M. La importancia de la investigación en odontología. J Oral Res. 2012;1(2):66.

12. Morales-Chávez M. La investigación y difusión de la ciencia odontológica en Venezuela y Latinoamérica. Odous Científica. 2016;17(1):41-48.

13. Castro A. Aprendizaje de la investigación en estudiantes universitarios [tesis doctoral]. Caracas (Venezuela): Universidad Nacional Experimental Simón Rodríguez; 2003.

14. Gutiérrez JM. La importancia de fortalecer la generación y la transmisión de conocimiento científico-tecnológico en Odontología. ODOVTOS-Int J Dent Sc. 2016;18(1):9-12.
15. Morales OA, Rincón ÁG, Romero JT. Cómo enseñar a investigar en la universidad. EDUCERE. Foro Universitario. 2005;9(29):217-224.

16. Cruz Santos RB, Torres G. Necesidades y características académicas laborales de los docentes que enseñan a investigar en la Facultad de Odontología de la UNAH, durante el tercer periodo de 2014. Portal de la Ciencia. 2015;8:95-119.

17. Fog L. Ranking revela universidades líderes en Latinoamérica. [Internet]. Sci Dev Net. 2010 [citado 01 de octubre 2017]. Disponible en: http://www.scidev.net/ america-latina/educacion/noticias/ranking-revela-universidades-l-deres-en-latinoam-rica-.html.

18. Gil JMS. Docencia e investigación en la universidad: una profesión, dos mundos. Educar. 2001;28:41-60.

19. Medina D. La investigación como vía de desarrollo para la labor docente en el nivel de educación media general. Mucutíes Universitaria. 2016;2:4-12.

20. Sarthou N. ¿Que veinte años no es nada?: Cambios y continuidades en los criterios de evaluación del Programa de Incentivos a Docentes-Investigadores en la universidad argentina (1994-2014). Rev iberoam cienc tecnol soc. 2016;11(32):85-110.

21. Campos N. El docente investigador: su génesis teórica y sus rasgos. Educación. 2003;27(2):39-42.

22. Roig-Vila R. Tecnología, innovación e investigación en los procesos de enseñanza-aprendizaje. Barcelona: Editorial Octaedro; 2016.

23. Boschma J. Generación Einstein. Más listos, más rápidos y más sociables. Barcelona: Gestión 2000; 2008.

24. Roig-Vila R, Mondéjar L, Lledó GL. Redes sociales científicas. La Web social al servicio de la investigación. IJERI. 2015;5:170-183.

25. Brunsell E, Horejsi M. Social networking. The Science Teacher. 2010;77(1):12-13.

26. Grané M, Willem C. Web 2.0: nuevas formas de aprender y participar. Barcelona: Laertes educación; 2008. 